\title{
Alleviative Effects of $\gamma$-Aminobutyric Acid (GABA) on Behavioral Abnormalities in Aged Dogs
}

\author{
Kentaro INAGAWA ${ }^{1)}$, Shinobu SEKI ${ }^{1)}$, Makoto BANNAI ${ }^{1}$, Yukari TAKEUCHI $^{2}$, Yuji MORI ${ }^{2}$ and Michio \\ TAKAHASHI ${ }^{1)}$ \\ ${ }^{1)}$ Research Institute for Health Fundamentals, Ajinomoto Co., Inc., 1-1 Suzukicho, Kawasaki-ku, Kawasaki-shi 210-8681 and \\ ${ }^{2}$ Laboratory of Veterinary Ethology, Graduate School of Agricultural and Life Sciences, The University of Tokyo 1-1-1 Yayoi, Bunkyo-ku, \\ Tokyo 113-8657, Japan
}

(Received 18 March 2005/Accepted 23 June 2005)

ABSTRACT. $\gamma$-aminobutyric acid (GABA, $30 \mathrm{mg} / \mathrm{kg}$ ) was administered to aged dogs with recent history of veterinary clinic visits (mean age: 15.3 years old) once daily for 2 weeks by mixing with food. Their owners subjectively evaluated the effects of GABA on behavioral signs often associated with aging in the dogs. Improvement in some of behavioral signs was notable without any observable adverse effects. Dogs administered with GABA tended to exhibit improvement in emotional states and signs may be caused by neurovegetable dysfunction, though effects on cognitive dysfunction syndrome were not always observed. Thus, GABA administration may be one of the effective means of improving the quality of life of aged dogs.

KEY WORDS: aged companion animals, behavioral signs, $\gamma$-aminobutyric acid.

J. Vet. Med. Sci. 67(10): 1063-1066, 2005

Recently, the lifespan of companion animals such as dogs and cats has been prolonged tremendously due to advances in veterinary medical care and the propagation of veterinary knowledge among the owners. A consequent increase in senile diseases in companion animals requires their owners' frequent and intensive care. Though common diseases such as cancer, cardiac and renal diseases are appropriately treated with the today's veterinary knowledge, therapeutic methods for the behavioral signs associated with aging or dementia have not yet been fully established.

The effect of oral administration of $\gamma$-aminobutyric acid (GABA) on severe human psychiatric disorders has previously been confirmed in observations on cases with various backgrounds such as children with mental retardation [8], juvenile delinquents with mental retardation consymptomed to a reformatory with medical facilities $[5,9]$, institutionalized psychiatric patients also suffering hypertension [1] and patients in a dementia state and with progressive paralysis [12]. Although effects on cognitive function were not always observed, improved emotional state e.g. greater stability (i.e. decrease in over-excitation) was evident. Together with that, improved spontaneity and capacity to carry out routine activities were also observed. Improvement in autistic behavior and depressive tendencies was observed as well.

Inspired by these observations of human studies, alleviative effects of GABA on behavioral signs in aged companion dogs were investigated preliminarily.

Aged dogs that were taken to veterinary hospitals (Imoto Animal Hospital, Kogure Animal Hospital, Senri New Town Animal Hospital, Takebe Veterinary Hospital, Tatematsu Veterinary Hospital, Uno Veterinary Hospital, Veterinary Hospital in Tokyo University, and Veterinary Surgical Service) and that showed clinical signs in terms of behavior were subjected to the present investigation. The dogs were selected according to the veterinarians' discretion, with requirements that there be signs to which behavioral modification techniques are difficult to apply and that the owner gives consent. The owner's consent was obtained by a formatted document. Prior to the test, the owners received information about the safety of the test preparation employed, and the owner's freedom to withdraw from the study at anytime was also assured. Such procedure resulted in testing 11 dogs with the mean age of 15.3 years old. The age range was between 12 and 19 years.

Test preparation contained GABA fine crystals (15\%), lactose $(38 \%)$, tapioca-starchy $(25 \%)$, dextrin $(20 \%)$ and $\mathrm{Ca}\left(\mathrm{PO}_{4}\right)_{2}(2 \%)$ was used. A $30 \mathrm{mg} / \mathrm{kg}$ body weight dose of this preparation was given once daily for 2 weeks by mixing with a regular meal or a snack (when a full meal was not given). No toxicity had been observed at this dose level during a 2-week sub-acute toxicity study that had been conducted using Beagle dogs reared for experimentation $(n=8$, body weight $9.5-10.7 \mathrm{~kg}$ ).

Behavioral abnormalities often associated with aging dogs were categorized into 10 clinical signs and graded on a 5-point scale (Table 1). The owner made the observation and evaluation of the behavioral signs before and after giving GABA. If 3 points or more were given to a dog with regard to a sign before receiving GABA, the dog was classified as a case exhibiting that sign. If the score of such a sign lowered after receiving GABA, the case was classified as "improved".

The effect of GABA with regard to a particular sign was assessed based on the proportion of improved cases (PIC, ranging from 0.0 to 1.0$)$, and rated tentatively into 3 grades; effective (PIC $>0.5)$, moderately effective $(0.2<\mathrm{PIC} \leq 0.5)$, and ineffective (PIC $\leq 0.2)$.

Additionally, optional subjective impressions were obtained from the owners concerning the behavioral state of 
Table 1. The format for the description of dog background and questionnaires for evaluation of therapeutic effect

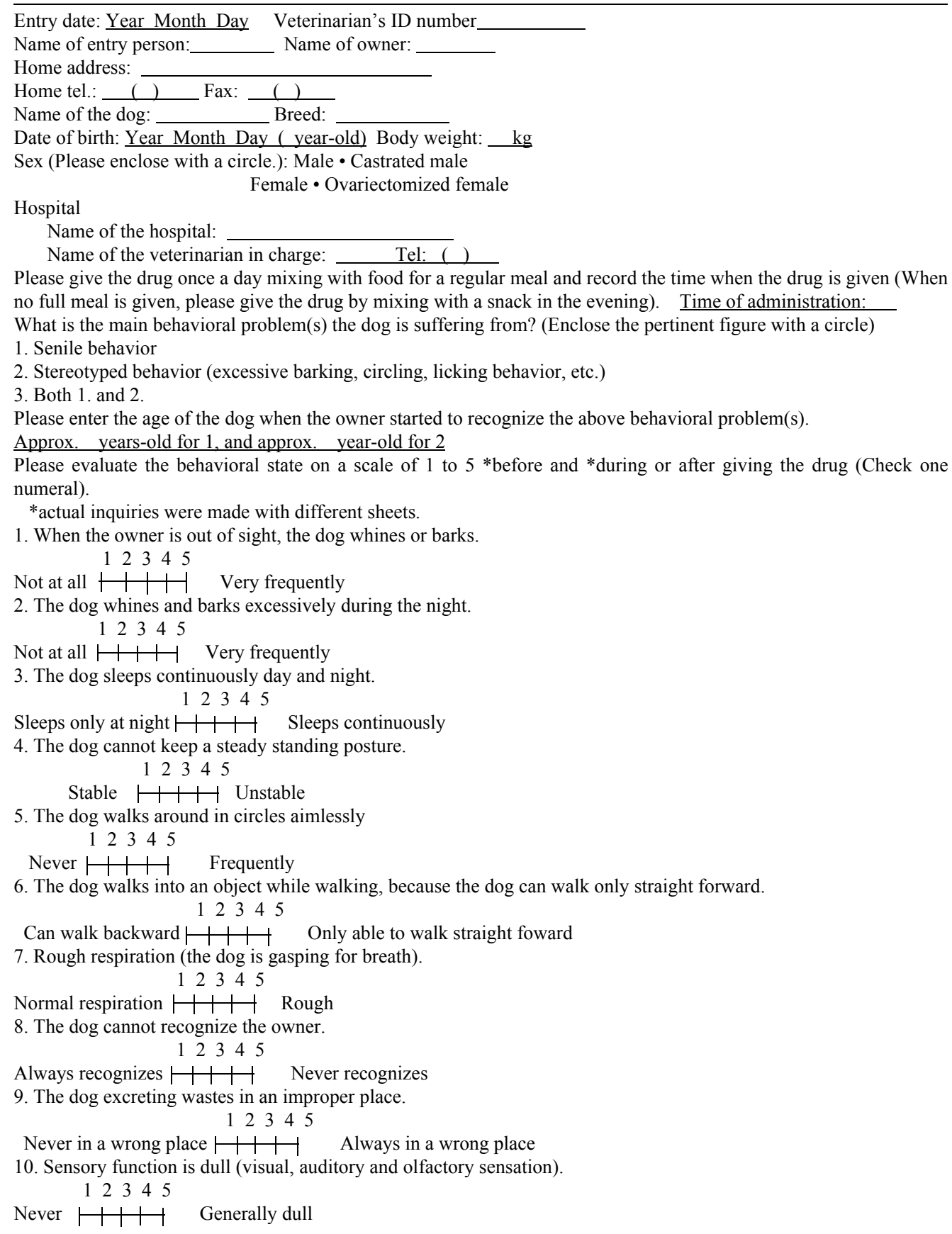

the dog during and after receiving GABA.

During the study period, no adverse effect was observed in any of the dogs. Changes in the grading scores of the 10 classified signs after the GABA administration are summarized in Table 2 .

The sign effectively alleviated by GABA (PIC > 0.5) was "frequent whines and barks during night" $(\mathrm{PIC}=0.67)$. The signs moderately alleviated $(0.2<\mathrm{PIC} \leq 0.5)$ were "whines and barks when left alone" (PIC $=0.5)$, “continual sleep dur- ing day and night" $(\mathrm{PIC}=0.25)$, "walking round without particular intention" $(\mathrm{PIC}=0.29)$ and "rough respiration" $(\mathrm{PIC}=0.5)$. The signs not effectively alleviated $(\mathrm{PIC} \leq 0.2)$ were "does not stand normally" $(\mathrm{PIC}=0)$, "failure to dodge objects" $(\mathrm{PIC}=0.14)$, "does not recognize owner" $(\mathrm{PIC}=0.2)$, "excretion of wastes at wrong places" $(\mathrm{PIC}=0.17)$, and "dull visual, auditory and olfactory sensation" (PIC=0.1).

Subjective impressions freely contributed from owners 
Table 2. Effects of GABA on senile behavioral signs

\begin{tabular}{llcc}
\hline & Improvement & $\begin{array}{c}\text { Number of } \\
\text { relevant } \\
\text { cases }\end{array}$ & $\begin{array}{c}\text { Proportion } \\
\text { of improved } \\
\text { cases }\end{array}$ \\
\hline Behavioral signs & 1 & & \\
1. When the owner is out of sight, the dog whines & $2^{\text {b) }}$ & 0.50 \\
$\quad$ and barks & 2 & $3^{\text {b) }}$ & 0.67 \\
2. The dog whines and barks during the night. & 2 & $8^{\text {b) }}$ & 0.25 \\
3. The dog sleeps continuously day and night. & 0 & $6^{\text {a) }}$ & 0.00 \\
4. The dog cannot keep a normal standing posture & 2 & $7^{\text {a) }}$ & 0.29 \\
5.The dog walks around in circles & 1 & $7^{\text {a) }}$ & 0.14 \\
6. The dog growls when hitting upon something, & 3 & $6^{\text {a) }}$ & 0.50 \\
because the dog can only walk straight. & 1 & $5^{\text {b) }}$ & 0.20 \\
7. Rough respiration & 1 & $6^{\text {c) }}$ & 0.17 \\
8. The dog cannot recognize its owner & 1 & $10^{\text {a) }}$ & 0.10 \\
9. The dog excretes wastes in a wrong place & &
\end{tabular}

The total number of 11(a), 10 (b), or 9(c) dogs were used.

concerning the behavioral state of the dog during and after receiving GABA were following 6 cases:

Mongrel A (17 years): Meaningless barking and dyspnea almost completely disappeared.

Mongrel B (14 years): Trembling in the legs was reduced and the dog was able to drink water without any help.

Toy poodle A (15 years): Dyspnea considerably improved, and indifference toward the owner's presence or calling was much reduced.

Toy poodle B (12 years): The dog had often staggered before the test, but now walks much more steadily. Meaningless barking decreased.

Pomeranian A (14 years): Lethargic state was much improved, and the dog now takes walks willingly.

Shiba A (16 years): Generally, the dog behaved more actively.

The present observations as a whole persuasively suggest that GABA administration once a day at a dose of $30 \mathrm{mg} / \mathrm{kg}$ body weight is beneficial for enhancing the quality of life in aged dogs suffering from various behavioral signs. Due to the insufficient number of cases, statistical analysis could not be applied to the present data. Thus, we tentatively categorized the effect into 3 grades based on the percentage of cases where there was beneficial effect.

It may be worth noting that the effectiveness on the "behavioral and/or mental disorder" has been reported in human studies, though the human participants were not necessarily aged $[1,5,8,9,12]$. The alleviative and moderating effects of GABA on the behavioral signs observed in the dogs were largely consistent with the effects observed in human studies. In addition, the present test results regarding cognitive dysfunction syndrome in dogs are also consistent with studies of human patients, both showing ineffectiveness of GABA on cognitive dysfunctions. This observation suggests that the effects of GABA are different from those of deprenyl, the MAO-B inhibitor [7].

We have previously shown that excitement of the neural structure located in the ventromedial nucleus of the hypo- thalamus (VMH) induces stereotyped running activity in rats [10], together with a rise of sympathetic tension accompanying the running [3]. Furthermore, in addition to the involvement of $\mathrm{VMH}$ in streotyped running, it has been elucidated that $\mathrm{VMH}$ controls the autonomic nervous system [11]. It is also widely known that the amygdala nucleus is an important part of the brain for the expression of emotion [4], and the neural connection between the $\mathrm{VMH}$ and the amygdala nucleus is well documented [2]. Improvement in behavior observed in the present study may be due to factors affecting the VMH via the neuronal network. And this running can be inhibited by GABA administration [6].

Thus, if some of the behavioral signs in aged dogs are attributable to insufficient inhibitory control of the VMH by GABA due to senescence, improvement of the signs observed in this study could be explained in this context, at least in part.

Any improvement of behavioral signs in aged companion animals is beneficial, since it lessens owners' labor and worry. This study strongly suggests, though more controlled trials are required, that GABA administration has beneficial effects, improving the quality of life of aged dogs.

The authors would like to express their gratitude to Dr. F. Imoto (Imoto Animal Hospital), Dr. N. Kogure (Kogure Animal Hospital), Dr. S. Matsunaga, Prof. H. Ogawa (Veterinary Hospital in Tokyo University), Dr. S. Sato (Senri New Town Animal Hospital), Dr. H. Takakura (Veterinary Surgical Service), Dr. M. Tatematsu (Tatematsu Veterinary Hospital) and Dr. T. Uno (Uno Veterinary Hospital) for their valuable discussions and collaboration in collecting data.

\section{REFERENCES}

1. Asano, M., Morisaki, H., Ishida, K., Matsutani, T., Hanashima, H. and Hayakawa, H. 1960. Kyosei Igaku 9: 97-102.

2. Carteras, N.S., Simerly, R.B. and Swanson, L.W. 1994. J. Comp. Neurol. 348: 41-79. 
3. Hirasawa, M., Nishihara, M. and Takahashi, M. 1996. Physiol. Behav. 59: 1017-1023.

4. LeDoux, J.E. 1996. The Emotional Brain-The Mysterious Underpinnings of Emotional Life. Simon \& Schuster, New York.

5. Mikami, K., Arai, S. and Harayama, A. 1960. Kyosei Igaku 9: 103-107.

6. Narita, K., Nishihara, M. and Takahashi, M. 1994. Brain Res. 642: 290-296.

7. Ruehl, W.W., Neilson, J., Hart, B., Head, E., Bruyette, D.S. and Cummings, B.J. 1998. Adv. Phamacol. 42: 316-319.

8. Takagi, R. and Maeda, M. 1959. Seishin Igaku 1: 811-820.

9. Tani, S. and Kuramochi, H. 1959. Seishin Igaku 1: 821-826.

10. Yokawa, T., Mitsushima, D., Itoh, C., Konishi, H., Shiota, K. and Takahashi, M. 1989. Physiol. Behav. 46: 713-717.

11. Yokawa, K., Shiota, K. and Takahashi, M. 1990. Physiol. Behav. 47: 1261-1264.

12. Yoshimura, Y., Ito, K. and Watanabe, H. 1962. Seishin Igaku 4: 565-570. 